\title{
A NEW SUBCLASS OF UNIVALENT FUNCTIONS
}

\section{GURMEET SINGH, GAGANDEEP SINGH, GURCHARANJIT SINGH}

\begin{abstract}
Complex analysis is an old and vulnerable subject. Geometric function theory is a branch of complex analysis that deals and studies the geometric properties of the analytic functions. The geometric function theory studies the classes of analytic functions in a domain lying in the complex plane $C$ subject to various conditions. The cornerstone of the Geometric function theory is the theory of univalent and multivalent functions which is considered as one of the active fields of the current research. Most of this field is concerned with the class $S$ of functions analytic and univalent in the unit disc $E=\{z:|z|<1\}$. One of the most famous problem in this field was Bieberbach Conjecture. For many years this problem stood as a challenge to the mathematicians and inspired the development of many new techniques in complex analysis. In the course of tackling Bieberbach Conjecture, new classes of analytic and univalent functions such as classes of convex and starlike functions were defined and some nice properties of these classes were widely studied. In the present study, we introduce an interesting subclass of analytic and close-to-convex functions in the open unit disc $E$. For functions belonging to this class, we derive several properties such as coefficient estimates, distortion theorems, inclusion relation, radius of convexity and Fekete-Szegö Problem. The various results presented here would generalize some known results.
\end{abstract}

Keywords: Subordination, univalent functions, starlike functions, close-to-convex functions, coefficient estimates, Fekete-Szegö problem

Mathematics Subject Classification: 30C45, 30C50

\section{INTRODUCTION}

Let $A$ be the class of functions of the form

$$
f(z)=z+\sum_{n=2}^{\infty} a_{n} z^{n}
$$

analytic in the open unit disc $E=\{z:|z|<1\}$. Let $S$ be the class of functions $f \in A$ and univalent in $E$.

By $U$ we denote the class of bounded or Schwarz functions $w(z)$ satisfying $w(0)=0$ and $|w(z)| \leqslant 1$ analytic in the unit disc $E$ and being of the form

$$
w(z)=\sum_{n=1}^{\infty} c_{n} z^{n}, z \in E .
$$

A function $f \in A$ is said to belong to the class $S^{*}$ of starlike functions if it satisfies the inequality:

$$
\operatorname{Re}\left(\frac{z f^{\prime}(z)}{f(z)}\right)>0(z \in E) .
$$

Gurmeet Singh, Gagandeep Singh, Gurcharanjit Singh, A New Subclass Of Univalent FuncTIONS.

(c) Gurmeet Singh, Gagandeep Singh, Gurcharanjit Singh 2019.

Submitted January 2, 2018. 
A function $f \in A$ is said to belong to the class $K$ of convex functions if it satisfies the inequality:

$$
\operatorname{Re}\left(\frac{\left(z f^{\prime}(z)\right)^{\prime}}{f^{\prime}(z)}\right)>0(z \in E) .
$$

A function $f \in A$ is said to belong to the class $C$ of close-to-convex if there exists a function $g \in S^{*}$ satisfying the condition:

$$
\operatorname{Re}\left(\frac{z f^{\prime}(z)}{g(z)}\right)>0(z \in E) .
$$

The concept of close-to-convex functions was introduced by Kaplan [4].

A function $f \in A$ is said to be starlike with respect to symmetric points in $E$ if it satisfies the condition:

$$
\operatorname{Re}\left(\frac{z f^{\prime}(z)}{f(z)-f(-z)}\right)>0 .
$$

This class is denoted by $S_{s}^{*}$ and was introduced and studied by Sakaguchi [10].

Since $\frac{f(z)-f(-z)}{2}$ is a starlike function in $E[1]$, the class $S_{s}^{*}$ also belongs to $C$.

Let $f$ and $g$ be two analytic functions in $E$. Then $f$ is said to be subordinate to $g$ (symbolically $f \prec g$ ) if there exists a bounded function $w(z) \in U$ such that $f(z)=g(w(z))$. This result is known as the principle of subordination.

In many earlier studies, various interesting subclasses of the analytic functions of class $A$ and the univalent functions of class $S$ were studied from a number of different view points. We choose to recall here some studies which are closely related to our work.

Following the concept of the class $S_{s}^{*}$, Gao and Zhou [2] discussed the following subclass of analytic functions, which is indeed a subclass of close-to-convex functions: Let $K_{S}$ denote the class of functions of the form (1) and satisfying the condition

$$
\operatorname{Re}\left(-\frac{z^{2} f^{\prime}(z)}{g(z) g(-z)}\right)>0
$$

where $g \in S^{*}\left(\frac{1}{2}\right)$.

Later, Kowalczyk and Les-Bomba [7] extended the class $K_{S}$ by introducing the following subclass of analytic functions:

A functions $f \in A$ is said to be in the class $K_{S}(\gamma), 0 \leqslant \gamma<1$, if there exists a function $g \in S^{*}\left(\frac{1}{2}\right)$ such that

$$
\operatorname{Re}\left(-\frac{z^{2} f^{\prime}(z)}{g(z) g(-z)}\right)>\gamma
$$

Obviously, $K_{S}(0) \equiv K_{S}$.

Recently Prajapat [9] introduced the following subclass of analytic functions:

A function $f \in A$ is said to be in the class $\chi_{t}(\gamma),|t| \leqslant 1, t \neq 0,0 \leqslant \gamma<1$, if there exists a function $g \in S^{*}\left(\frac{1}{2}\right)$ such that

$$
\operatorname{Re}\left(\frac{t z^{2} f^{\prime}(z)}{g(z) g(t z)}\right)>\gamma
$$

In particular, $\chi_{-1}(\gamma) \equiv K_{S}(\gamma)$ and $\chi_{-1}(0) \equiv K_{S}$.

Motivated by the above defined classes, we introduce the following subclass of analytic functions: 
Let $\chi_{t}(A, B),|t| \leqslant 1, t \neq 0$, denote the class of functions $f \in A$ and satisfying the conditions

$$
\frac{t z^{2} f^{\prime}(z)}{g(z) g(t z)} \prec \frac{1+A z}{1+B z}, \quad-1 \leqslant B<A \leqslant 1, \quad z \in E
$$

where $g \in S^{*}\left(\frac{1}{2}\right)$.

The following observations are obvious:

(i) $\chi_{t}(1-2 \gamma,-1) \equiv \chi_{t}(\gamma)$.

(ii) $\chi_{-1}(1-2 \gamma,-1) \equiv K_{S}(\gamma)$.

(iii) $\chi_{-1}(1,-1) \equiv K_{S}$.

By definition of subordination it follows that $f \in \chi_{t}(A, B)$ if and only if $f(z)$ can be represented as

$$
\frac{t z^{2} f^{\prime}(z)}{g(z) g(t z)}=\frac{1+A w(z)}{1+B w(z)}, \quad w(z) \in U, \quad-1 \leqslant B<A \leqslant 1, \quad z \in E .
$$

In the present work, we obtain the coefficient estimates, inclusion relation, distortion theorems, radius of convexity and Fekete-Szegö problem for the functions in the class $\chi_{t}(A, B)$. Our results extend the known results due to various authors.

Throughout our present discussion, to avoid repetition, we lay down once for all that

$$
-1 \leqslant B<A \leqslant 1, \quad 0<|t| \leqslant 1, \quad t \neq 0, \quad z \in E .
$$

\section{MAIN Results}

2.1. Estimates for coefficients. To prove the results in this subsection, we make use of the following lemmata.

Lemma $1(\sqrt{3} \mid)$. Let

$$
\frac{t z^{2} f^{\prime}(z)}{g(z) g(t z)}=P(z)=1+\sum_{n=1}^{\infty} p_{n} z^{n}
$$

then

$$
\left|p_{n}\right| \leqslant(A-B), n \geqslant 1 \text {. }
$$

The bounds are sharp being attained at the functions

$$
P_{n}(z)=\frac{1+A \delta z^{n}}{1+B \delta z^{n}}, \quad|\delta|=1 .
$$

Lemma 2 ([1] $)$. As $g \in S^{*}\left(\frac{1}{2}\right)$, for

$$
G(z)=\frac{g(z) g(t z)}{t z}=z+\sum_{n=2}^{\infty} d_{n} z^{n} \in S^{*},
$$

we have $\left|d_{n}\right| \leqslant n$.

Theorem 1. If $f \in \chi_{t}(A, B)$, then

$$
\left|a_{n}\right| \leqslant 1+\frac{(n-1)(A-B)}{2} .
$$

Proof. As $f \in \chi_{t}(A, B)$, we can express (5) as

$$
\frac{z f^{\prime}(z)}{G(z)}=P(z)
$$


Using (1), (6) and (8) in (10), we get

$$
1+\sum_{n=2}^{\infty} n a_{n} z^{n-1}=\left(1+\sum_{n=2}^{\infty} n d_{n} z^{n-1}\right)\left(1+\sum_{n=1}^{\infty} p_{n} z^{n}\right) .
$$

Equating the coefficients of $z^{n-1}$ in $(11)$, we have

$$
n a_{n}=d_{n}+d_{n-1} p_{1}+d_{n-2} p_{2}+\ldots+d_{2} p_{n-2}+p_{n-1} .
$$

Therefore. using Lemma 1 and Lemma 2, we get

$$
n\left|a_{n}\right| \leqslant n+(A-B)[(n-1)+(n-2)+\ldots+2+1] .
$$

Hence, by (13), we easily obtain (9).

Letting $A=1-2 \gamma, B=-1$ in Theorem 1, the following result due to Prajapat [9] becomes obvious.

Corollary 1. If $f \in \chi_{t}(\gamma)$, then

$$
\left|a_{n}\right| \leqslant 1+(n-1)(1-\gamma) .
$$

2.2. Inclusion relation. The following lemma is useful in the proof of the main result in this subsection.

Lemma 3 ([11]). Let

$$
-1 \leqslant B_{2} \leqslant B_{1}<A_{1} \leqslant A_{2} \leqslant 1,
$$

then

$$
\frac{1+A_{1} z}{1+B_{1} z} \prec \frac{1+A_{2} z}{1+B_{2} z} .
$$

Theorem 2. Let

$$
-1 \leqslant B_{2} \leqslant B_{1}<A_{1} \leqslant A_{2} \leqslant 1,
$$

then

$$
\chi_{t}\left(A_{1}, B_{1}\right) \subset \chi_{t}\left(A_{2}, B_{2}\right) .
$$

Proof. As $f \in \chi_{t}\left(A_{1}, B_{1}\right)$, therefore

$$
\frac{t z^{2} f^{\prime}(z)}{g(z) g(t z)} \prec \frac{1+A_{1} z}{1+B_{1} z} .
$$

Since

$$
-1 \leqslant B_{2} \leqslant B_{1}<A_{1} \leqslant A_{2} \leqslant 1,
$$

by Lemma 3, we have

$$
\frac{t z^{2} f^{\prime}(z)}{g(z) g(t z)} \prec \frac{1+A_{1} z}{1+B_{1} z} \prec \frac{1+A_{2} z}{1+B_{2} z} .
$$

This yields that $f \in \chi_{t}\left(A_{2}, B_{2}\right)$ and this proves the inclusion relation.

\subsection{Distortion theorems.}

Theorem 3. If $f \in \chi_{t}(A, B)$, then for $|z|=r, 0<r<1$, we have

$$
\frac{(1-A r)}{(1-B r)(1+r)^{2}} \leqslant\left|f^{\prime}(z)\right| \leqslant \frac{(1+A r)}{(1+B r)(1-r)^{2}}
$$

and

$$
\int_{0}^{r} \frac{(1-A t)}{(1-B t)(1+t)^{2}} d t \leqslant|f(z)| \leqslant \int_{0}^{r} \frac{(1+A t)}{(1+B t)(1-t)^{2}} d t .
$$


Proof. From (10), we have

$$
\left|f^{\prime}(z)\right|=\frac{|G(z)|}{|z|}\left|\frac{1+A w(z)}{1+B w(z)}\right|, w(z) \in B .
$$

It is easy to show that the transform

$$
\frac{z f^{\prime}(z)}{G(z)}=\frac{1+A w(z)}{1+B w(z)}
$$

maps $|w(z)| \leqslant r$ onto the circle

$$
\left|\frac{z f^{\prime}(z)}{G(z)}-\frac{1-A B r^{2}}{1-B^{2} r^{2}}\right| \leqslant \frac{(A-B) r}{\left(1-B^{2} r^{2}\right)},|z|=r .
$$

This implies that

$$
\frac{1-A r}{1-B r} \leqslant\left|\frac{1+A w(z)}{1+B w(z)}\right| \leqslant \frac{1+A r}{1+B r} .
$$

Since by Lemma 2, $G(z)$ is a starlike function and so due to a well known result, we have

$$
\frac{r}{(1+r)^{2}} \leqslant|G(z)| \leqslant \frac{r}{(1-r)^{2}} .
$$

Equation (16) together with (17) and (18) yields (14). On integrating (14) from 0 to $r$, (15) follows.

For $A=1-2 \gamma, B=-1$, Theorem 3 gives the following result due to Prajapat [9]:

Corollary 2. If $f \in \chi_{t}(\gamma)$, then

$$
\frac{1-(1-2 \gamma) r}{(1+r)^{3}} \leqslant\left|f^{\prime}(z)\right| \leqslant \frac{1+(1-2 \gamma) r}{(1-r)^{3}}
$$

and

$$
\int_{0}^{r} \frac{1-(1-2 \gamma) t}{(1+t)^{3}} d t \leqslant|f(z)| \leqslant \int_{0}^{r} \frac{1+(1-2 \gamma) t}{(1-t)^{3}} d t .
$$

\subsection{Radius of convexity.}

Theorem 4. If $f \in \chi_{t}(A, B)$, then $f(z)$ is convex in $|z|<r_{1}$, where $r_{1}$ is the smallest positive root in $(0,1)$ of the equation

$$
A B r^{3}-A(B-2) r^{2}-(2 B-1) r-1=0 .
$$

Proof. As $f \in \chi_{t}(A, B)$, we have

$$
z f^{\prime}(z)=G(z) p(z)
$$

After logarithmic differentiating (20), we get

$$
1+\frac{z f^{\prime \prime}(z)}{f^{\prime}(z)}=\frac{z G^{\prime}(z)}{G(z)}+\frac{z p^{\prime}(z)}{p(z)} .
$$

Now for $G(z) \in S^{*}$ we have

$$
\operatorname{Re}\left(\frac{z G^{\prime}(z)}{G(z)}\right) \geqslant \frac{1-r}{1+r}
$$

Therefore, 21 yields that

$$
\operatorname{Re}\left(1+\frac{z f^{\prime \prime}(z)}{f^{\prime}(z)}\right) \geqslant \frac{1-r}{1+r}-\left|\frac{z p^{\prime}(z)}{p(z)}\right| .
$$

Further, we have

$$
\operatorname{Re}\left(1+\frac{z f^{\prime \prime}(z)}{f^{\prime}(z)}\right) \geqslant \frac{1-r}{1+r}-\frac{r(A-B)}{(1+A r)(1+B r)} .
$$


After simplification we obtain

$$
\operatorname{Re}\left(1+\frac{z f^{\prime \prime}(z)}{f^{\prime}(z)}\right) \geqslant \frac{-A B r^{3}+A(B-2) r^{2}+(2 B-1) r+1}{(1+r)(1+A r)(1+B r)} .
$$

Hence, the function $f(z)$ is convex in $|z|<r_{1}$, where $r_{1}$ is the smallest positive root in $(0,1)$ of the equation

$$
A B r^{3}-A(B-2) r^{2}-(2 B-1) r-1=0 .
$$

For $A=1-2 \gamma, B=-1$, Theorem 4 gives the following result by Prajapat [9]:

Corollary 3. If $f \in \chi_{t}(\gamma)$, then $f(z)$ is convex in $|z|<r_{0}=2-\sqrt{3}$.

2.5. Fekete-Szegö Problem. We use the following lemmata to prove the results in this subsection:

Lemma 4. ([5], [8]) If $p(z)=1+p_{1} z+p_{2} z^{2}+p_{3} z^{3}+\ldots$ is a function with positive real part, then for each complex number $\mu$,

$$
\left|p_{2}-\mu p_{1}^{2}\right| \leqslant 2 \max \{1,|2 \mu-1|\}
$$

and the result is sharp for the functions given by

$$
p(z)=\frac{1+z^{2}}{1-z^{2}}, \quad p(z)=\frac{1+z}{1-z} .
$$

Lemma $5([6])$. If

$$
G(z)=z+\sum_{n=2}^{\infty} d_{n} z^{n} \in S^{*},
$$

then for each complex number $\lambda$ obeying $\left|d_{3}-\lambda d_{2}^{2}\right| \leqslant \max \{1,|3-4 \lambda|\}$ and the result is sharp for the Koebe function $k$ if

$$
\left|\lambda-\frac{3}{4}\right| \geqslant \frac{1}{4}
$$

and for

$$
k^{\frac{1}{2}}\left(z^{2}\right)=\frac{z}{1-z^{2}}
$$

if

$$
\left|\lambda-\frac{3}{4}\right| \leqslant \frac{1}{4}
$$

Theorem 5. If $f \in \chi_{t}(A, B)$, then for $\mu \in \mathbb{C}$ we have

$$
\left|a_{3}-\mu a_{2}^{2}\right| \leqslant \frac{(A-B)}{3} \max \left\{1,\left|2 \gamma_{1}-1\right|\right\}+\frac{1}{3} \max \left\{1,\left|3-4 \mu_{1}\right|\right\}+2(A-B)\left|\frac{1}{3}-\frac{\mu}{2}\right|,
$$

where

$$
\gamma_{1}=\frac{(1+B)}{2}+\frac{3(A-B) \mu}{8}, \quad \mu_{1}=\frac{3 \mu}{4} .
$$

Proof. As $f \in \chi_{t}(A, B)$, by (5) we have

$$
\frac{z f^{\prime}(z)}{G(z)}=\frac{1+A w(z)}{1+B w(z)}
$$

Let

$$
h(z)=\frac{1+w(z)}{1-w(z)}=1+p_{1} z+p_{2} z^{2}+p_{3} z^{3}+\ldots,
$$


then $\operatorname{Re}(h(z))>0$ and $h(0)=1$. Hence,

$$
\frac{z f^{\prime}(z)}{G(z)}=\frac{1-A+h(z)(1+A)}{1-B+h(z)(1+B)}
$$

We expanding $(23)$ to obtain

$$
\begin{aligned}
1+\left(2 a_{2}-d_{2}\right) z+\left(3 a_{3}-2 a_{2} d_{2}-d_{3}+d_{2}^{2}\right) z^{2}+\ldots=1 & +\frac{p_{1}(A-B) z}{2} \\
& +\frac{(A-B)}{2}\left(p_{2}-p_{1}^{2}\left(\frac{1+B}{2}\right)\right) z^{2}+\ldots
\end{aligned}
$$

Equating the coefficients at $z$ and $z^{2}$ on both sides of the above equation, we get

$$
a_{2}=\frac{2 d_{2}+p_{1}(A-B)}{4}
$$

and

$$
a_{3}=\frac{1}{3}\left(d_{3}+\frac{(A-B)}{2}\left(p_{1} d_{2}+p_{2}-\frac{p_{1}^{2}(1+B)}{2}\right)\right) .
$$

Therefore, we have

$$
\left|a_{3}-\mu a_{2}^{2}\right| \leqslant \frac{(A-B)}{6}\left|p_{2}-\gamma_{1} p_{1}^{2}\right|+\frac{\left|d_{3}-\mu_{1} d_{2}^{2}\right|}{3}+\frac{(A-B)}{2}\left|d_{2}\right|\left(\frac{1}{3}-\frac{\mu}{2}\right)\left|p_{1}\right| .
$$

Using Lemma 4 and Lemma 5 , we complete the proof.

For $A=1-2 \gamma, B=-1$, Theorem 5 gives the following result.

Corollary 4 If $f \in \chi_{t}(\gamma)$, then for $\mu \in C$,

$$
\left|a_{3}-\mu a_{2}^{2}\right| \leqslant \frac{2(1-\gamma)}{3} \max \left\{1,\left|2 \gamma_{1}-1\right|\right\}+\frac{\max \left\{1,\left|3-4 \mu_{1}\right|\right\}}{3}+4(1-\gamma)\left|\frac{1}{3}-\frac{\mu}{2}\right|
$$

where

$$
\gamma_{1}=\frac{3(1-\gamma) \mu}{4}, \quad \mu_{1}=\frac{3 \mu}{4}
$$

\section{BIBLIOGRAPHY}

1. R.N. Das, P. Singh. On subclasses of schlicht mapping // Indian J. Pure Appl. Math. 8:8, 864-872 (1977).

2. C.Y. Gao, S.Q. Zhou. On a class of analytic functions related to the starlike functions // Kyungpook Math. J. 45:1, 123-130 (2005).

3. R.M. Goel, Beant Singh Mehrok. A subclass of univalent functions // Houston J. Math. 8:3, 343-357 (1982).

4. W. Kaplan, Close-to-convex schlicht functions // Michigan Math. J. 1:2, 169-185 (1952).

5. F.R. Keogh, E.P. Merkes, A coefficient inequality for certain class of analytic functions // Proc. Amer. Math. Soc. 20:1, 8-12 (1969).

6. W. Koepf. On the Fekete-Szegö problem for close-to-convex functions // Proc. Amer. Math. Soc. 10:1, 89-95 (1993).

7. J. Kowalczyk, E. Les-Bomba. On a subclass of close-to-convex functions // Appl. Math. Letters 23:10, 1147-1151 (2010).

8. R.J. Libera, E.J. Zlotkiewicz. Coefficient bounds for the inverse of a function with derivative in $\rho / /$ Proc. Amer. Math. Soc. 87:2, 251-257 (1983).

9. J.K. Prajapat. A new subclass of close-to-convex functions // Surv. Math. Appl. 11, 11-19 (2016).

10. K. Sakaguchi. On a certain univalent mapping // J. Math. Soc. Japan. 11:1, 72-75 (1959).

11. Amit Soni, Shashi Kant. A new subclass of close-to-convex functions with Fekete-Szegö problem // J. Rajasthan Acad. Phy. Sci. 12:2, 1-14, (2013). 
Gurmeet Singh,

Principal, Patel Memorial National College,

Rajpura-140401, Punjab, India

E-mail: meetgur111@gmail.com

Gagandeep Singh,

Assistant Professor, Department of Mathematics,

Majha College For Women,

Tarn Taran-143401, Punjab, India

E-mail: kamboj.gagandeep@yahoo.in

Gurcharanjit Singh,

Research Fellow, Department of Mathematics,

Punjabi University,

Patiala-147002, Punjab, India

E-mail: dhillongs82@yahoo.com 